low, irrespective of the sensitivity of available POC tests. In highprevalence settings, sufficient coverage and screening frequency must be maintained to avert an increase in infectious syphilis prevalence.

\section{P1-S6.27 COULD A PEER DRIVEN INTERVENTION INCREASE UPTAKE OF CHLAMYDIA SCREENING? PROOF OF PRINCIPLE}

\section{doi:10.1136/sextrans-2011-050108.251}

${ }^{1} \mathrm{P}$ Horner, ${ }^{2} \mathrm{~J}$ Loaring, ${ }^{1} \mathrm{H}$ Matthew, ${ }^{3} \mathrm{I}$ Oliver, ${ }^{1} \mathrm{R}$ Campbell, ${ }^{1} \mathrm{C}$ Trotter, ${ }^{1} \mathrm{~J}$ Macleod, ${ }^{4} \mathrm{~K}$ Pye. ${ }^{1}$ University of Birmingham, Birmingham, UK; ${ }^{2}$ University of Bristol, UK; ${ }^{3} \mathrm{Health}$ Protection Agency, UK; ${ }^{4}$ Bristol Primary Care Trust, UK

Background Uptake of the opportunistic National Chlamydia Screening Programme in England is low and below model estimates of the optimal level to reduce Chlamydia incidence. Peer led approaches may increase screening uptake but their feasibility and acceptability to young people is not known.

Methods Focus groups and interviews with young women and men. All participants had expressed an interest in undergoing Chlamydia screening or had already been screened. The focus group and interviews were audio taped and transcribed verbatim for analysis. Thematic analysis of the data was conducted to identify, compare and report patterns in the data. Following interview, Chlamydia postal kits were introduced to participants and their opinions on giving these out to their peers sought. Participants were asked for their views and experiences of discussing Chlamydia screening and distributing kits to their friends four to eight weeks after the focus group/interview. All kits returned over a 9-month period to the laboratory were recorded.

Results Six men (mean age 19 years) and 6 women (mean age 20 years) were recruited. In total 45 kits were distributed, $33(73 \%)$ to female participants. $26(79 \%)$ and $3(25 \%)$ of kits given to females and males respectively were given to peers. Of those tests distributed to peers there is a high return rate 34\% (10/29) all of which had been given out by females. On average, 1.7 kits were returned for each female participant. Participants generally felt positive about the idea of peer-driven screening using postal kits. However, embarrassment was a key theme, particularly among men. Generally women but not men were able to discuss Peer Driven Screening among their close friends. Both sexes felt Peer Driven Screening would be easier if kits were readily available in multiple sites, and Chlamydia screening was more widely promoted.

Conclusion Female Peer Driven Screening but not male Peer Driven Screening was successful in recruiting peers to participate in Chlamydia screening. A peer driven intervention may increase uptake of Chlamydia screening.

\section{P1-S6.28 INTEGRATED HIV, SYPHILIS, AND OTHER STI TESTING IN NORTH CAROLINA COUNTY JAILS}

doi:10.1136/sextrans-2011-050108.252

${ }^{1} \mathrm{~J}$ Scott, ${ }^{2} \mathrm{~L}$ A Sampson, ${ }^{2} \mathrm{~J} \mathrm{M}$ Clymore, ${ }^{1} \mathrm{P}$ R Moore, ${ }^{2} \mathrm{P}$ A Leone. ${ }^{1}$ Centers for Disease Control \& Prevention, Raleigh, USA; ${ }^{2}$ NC Division of Public Health, Raleigh, USA

Background In 1999 North Carolina initiated STI screening programs in seven county jails as part of the CDC Syphilis Elimination Effort (SEE). All jails were willing to permit screening programs for syphilis but only two also screened for HIV. In 2007 the SEE funding was depleted and the 7 SEE jail projects were converted to HIV screening under the CDC Expanded Testing Initiative (ETI). New jails were added $(n=21)$ and several have added screening for other STIs. Testing is supported by 10 local health departments and 5 non-traditional testing sites which assure treatment for STDs and make referrals for HIV care services.

Methods From January to June 2010, STI screening data for 28 jails was analysed. All jails tested for HIV and syphilis, one jail added HCV screening and another jail added NAAT testing for gonorrhoea and chlamydial infection. Reactive syphilis tests and positive HIV tests were verified against surveillance records to determine whether or not they were previous cases or newly diagnosed infections. Positive tests for Neisseria gonorrhoeae and Chlamydia trachomatis were considered to be new infections. Positive tests for HCV are considered prevalent infections. All data were analysed using SAS 9.1.3.

Results Under SEE, only two jails screened for both HIV and syphilis. From 2002 to 2005, 3626 inmates were screened. There were 46 (1.3\%) HIV-positive inmates and 158 (4.4\%) with reactive syphilis tests. Of those 158,30 were confirmed new syphilis cases $(0.83 \%$ case rate). Under ETI, 28 jails screened 4688 inmates for syphilis and 4662 for HIV in just 6 months (January-June, 2010, see Abstract P1-S6.28 table 1). HIV positivity rates were similar for men and women $(p=0.90)$ but women were more likely than men to be both a syphilis reactor (OR 4.8 95\% CI 2.8 to 8.2) and a new case (OR 5.9 95\% CI 1.9 to 17.9). Among all positive tests, there were no comorbid patients.

Abstract P1-S6.28 Table 1 HIV and STI testing in North Carolina County Jails, January-June, 2010

\begin{tabular}{|c|c|c|c|c|c|c|}
\hline \multirow[b]{2}{*}{ STI** } & \multicolumn{2}{|l|}{ Men } & \multicolumn{2}{|l|}{ Women } & \multicolumn{2}{|l|}{ All* } \\
\hline & Tested & Positive & Tested & Positive & Tested & Positive \\
\hline HIV & 3640 & $17(0.47 \%)$ & 1006 & $5(0.50 \%)$ & 4662 & $22(0.47 \%)$ \\
\hline New Cases*** & & $2(0.05 \%)$ & & $1(0.10 \%)$ & & $3(0.06 \%)$ \\
\hline Syphilis & 3633 & $24(0.66 \%)$ & 1000 & $31(3.10 \%)$ & 4688 & $55(1.18 \%)$ \\
\hline New Cases ${ }^{* * *}$ & & $5(0.14 \%)$ & & $8(0.80 \%)$ & & $13(0.28 \%)$ \\
\hline HCV & 201 & $11(5.47 \%)$ & 52 & $3(5.77 \%)$ & 253 & $14(5.53 \%)$ \\
\hline $\mathrm{Ct}$ & 202 & $14(6.93 \%)$ & 28 & 0 & 230 & 14 (6.09\%) \\
\hline Gc & 200 & 0 & 28 & $1(3.57 \%)$ & 228 & $1(0.44 \%)$ \\
\hline
\end{tabular}

*Includes transgender, missing sex. ${ }^{* *} \mathrm{n}=28$ jails tested for syphilis and HIV, 1 jail tested for $\mathrm{HCV}$ and a different jail tested for $\mathrm{Ct} / \mathrm{Gc}$. ${ }^{* *}$ New cases verified against surveillance data.

Conclusions The jail setting proves to be an important setting for detecting new syphilis cases, particularly among women, and for identifying HIV-positive inmates. Many of these HIV positives were not new diagnoses but may represent persons who need referrals for care and treatment. Screening for HCV and combined $\mathrm{Ct} / \mathrm{Gc}$ screening was also productive and should be expanded to other jails. Due to the high concentration of persons who trade sex for drugs or money in jail settings, it is especially important that these HIV and STI cases be identified and referred for treatment and partner services to prevent ongoing transmission.

\section{P1-S6.29 LONGITUDINAL TRENDS IN HIV TESTING AND PREVALENCE AMONG STI CLINIC PATIENTS IN LILONGWE, MALAWI: 2006-2010}

doi:10.1136/sextrans-2011-050108.253

${ }^{1} \mathrm{~K}$ Powers, ${ }^{2} \mathrm{G}$ Kamanga, ${ }^{2} \mathrm{C}$ Mapanje, ${ }^{2} \mathrm{~J} \mathrm{~K}$ Malava, ${ }^{2} \mathrm{M}$ Chindebvu, ${ }^{2} \mathrm{H}$ Kamzati, ${ }^{2} \mathrm{~F}$ Martinson, ${ }^{1} \mathrm{~W}$ Miller, 'M Cohen, ${ }^{1} \mathrm{I}$ Hoffman. ' University of North Carolina, Chapel Hill, USA; ${ }^{2}$ UNC Project Malawi, Lilongwe, Malawi

Background To understand longitudinal trends in HIV status awareness, HIV test acceptance, and HIV prevalence among 
patients of a sexually transmitted infections (STI) clinic in Lilongwe, Malawi, we conducted descriptive analyses of these endpoints over the last 5 years.

Methods Using data collected routinely at all STI clinic visits since March 2006, we calculated the proportion of visits in each of the last five calendar years $(2006,2007,2008,2009$, and 2010) during which patients reported a previous HIV test, and among those, the proportions reporting positive and negative results. We also calculated the proportion accepting HIV tests at the clinic, and among those who received HIV tests at their visits, the proportion who had positive results. We conducted log-binomial regression with generalised estimating equations to analyse trends in each endpoint over time.

Results An average of 8944 clinic visits occurred each year, approximately $60 \%$ by females and $40 \%$ by males. The proportion of visits at which patients reported a previous HIV test increased from $57 \%$ in 2006 to $86 \%$ in 2010 ( $p<0.0001$ ) (Abstract P1-S6.29 figure 1). Among those reporting a previous test, the proportions reporting negative or positive results remained relatively constant, at approximately $70 \%$ and $30 \%$, respectively (results not shown). At visits where patients did not report a prior positive HIV test, the proportion accepting HIV antibody tests increased from $50 \%$ to $78 \%(p<0.0001)$. Among all those who received HIV antibody tests at a visit, the proportion who were found to be HIV-positive decreased from $31 \%$ to $17 \%(p<0.0001)$, with a decrease from $13 \%$ to $10 \%$ among those reporting a previous negative test $(\mathrm{p}<0.0001)$ (Abstract P1-S6.29 figure 1)

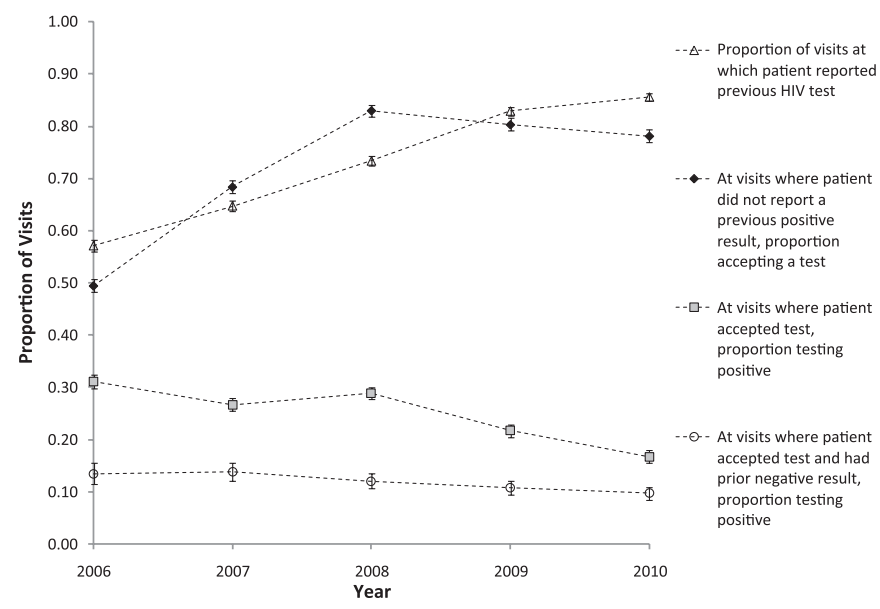

Abstract P1-S6.29 Figure 1 Longitudinal Trends in HIV Testing and HIV Prevalence, Kamuzu Central Hospital STI Clinic, Lilongwe, Malawi, 2006-2010.

Conclusions Over the last 5 years, the proportion of STI clinic visits at which patients reported awareness of their HIV status has increased, including an increase in known positives to $25 \%$ of all patients. At visits where patients did not report a previous positive result, the proportion who accepted rapid antibody tests has also increased. Among those receiving HIV tests at their clinic visits, the proportion testing HIV-positive has decreased substantially, although $10 \%$ of those with a previous negative result tested positive. These findings raise great concern about ongoing HIV acquisition and transmission in patients becoming aware of their serostatus through expanded HIV testing. Novel and better HIV prevention strategies for these patients are urgently needed.

\section{P1-S6.30 HIV TESTING OF PATIENTS RECEIVING AN STD EVALUATION IN A NORTH CAROLINA COMMUNITY HEALTH CENTER}

doi:10.1136/sextrans-2011-050108.254

${ }^{1} \mathrm{P}$ Klein, ${ }^{2} \mathrm{~A}$ Bishop, ${ }^{3} \mathrm{P}$ Leone. ${ }^{1}$ UNC Gillings School of Global Public Health, Chapel Hill, USA; ${ }^{2}$ Robeson Healthcare Corporation, USA; ${ }^{3}$ University of North Carolina School of Medicine, USA

Background The CDC has recommended HIV testing for persons with a suspected STD since 1987 and for all patients in clinical settings since 2006. However, HIV testing of suspected STD patients in emergency medical sites is inadequate, despite the high risk of HIV acquisition in this population. Community health centers may have better adherence to HIV testing recommendations due to a more personal relationship with their clientele and heightened awareness of state disease trends.

Methods Robeson Healthcare Corporation is a federally-qualified health center with 4 sites located in a county in the top $10 \%$ of HIV case rates and top $33 \%$ of syphilis case rates in North Carolina. A routine HIV testing program was started in June 2009. Patients receiving an STD evaluation 1 June 2009 through 31 January 2011 were examined for concurrent HIV testing. The association between age and HIV testing among STD testers was assessed with multivariate logistic regression with a robust variance estimator to account for patients with multiple clinic visits.

Results Of the 6588 clinic visits that included an STD evaluation, 2324 also included an HIV test (35.3\%, 95\% CI 32.1 to $36.4 \%$ ). Over $70 \%$ of patients tested for syphilis also received an HIV test ( $n=1531 / 2111,72.5 \%, 95 \%$ CI 70.6 to $74.4 \%$ ). Less than $30 \%$ of patients screened for gonorrhoea and Chlamydial infections were also tested for HIV ( $\mathrm{n}=1545 / 5442,28.4 \%$, 95\% CI 27.2 to $29.6 \%$ ). During a statewide syphilis outbreak in 2009 with high HIVsyphilis coinfection rates, HIV testing of suspected syphilis patients reached $84.0 \%$ (95\% CI 80.9 to $87.0 \%$ ). Patients of older ages were less likely to be tested for HIV during their STD evaluation than patients of younger ages (Abstract P1-S6.30 table 1).

Abstract P1-S6.30 Table 1 HIV Testing of patients receiving an STD Evaluation by Patient Age, June 2009-January 2011

\begin{tabular}{llll}
\hline & OR (95\% CI) & OR (95\% CI) & OR (95\% CI) \\
\hline $\begin{array}{llll}\text { Patient age (years) } \\
13-24\end{array}$ & 1.00 & 1.00 & 1.00 \\
$25-34$ & $0.76(0.59$ to 0.98$)$ & $0.81(0.71$ to 0.91$)$ & $0.45(0.21$ to 1.00$)$ \\
$35-44$ & $0.31(0.23$ to 0.42$)$ & $0.48(0.40$ to 0.58$)$ & $0.55(0.22$ to 1.37$)$ \\
$45-54$ & $0.16(0.11$ to 0.24$)$ & $0.29(0.22$ to 0.40$)$ & $0.23(0.07$ to 0.72$)$ \\
$55-64$ & $0.10(0.06$ to 0.20$)$ & $0.28(0.17$ to 0.44$)$ & $0.39(0.09$ to 1.75$)$ \\
$65+$ & $0.05(0.02$ to 0.11$)$ & $0.14(0.03$ to 0.59$)$ & $0.39(0.03$ to 4.61$)$ \\
Age (10-year interval) & $0.51(0.47$ to 0.57$)$ & $0.68(0.63$ to 0.72$)$ & $0.75(0.58$ to 0.98$)$ \\
\hline
\end{tabular}

Conclusions In this community health center population, over $70 \%$ of patient visits with a syphilis evaluation included a concurrent HIV test order. This represents an improvement from an emergency department setting, in which less than $30 \%$ of syphilis testers were also tested for HIV. However, HIV testing among patients screened for gonorrhoea and Chlamydial infection was still low; the inclusion of HIV testing in the standard order panel for patients receiving an STD evaluation may increase HIV testing in this highrisk population. 